\title{
Accurately measuring ice thickness quickly and quantitatively on a screening TEM
}

\author{
Hamish Brown ${ }^{1}$ and Eric Hanssen ${ }^{2}$
}

${ }^{1}$ The University of Melbourne, Parkville, Victoria, Australia, ${ }^{2}$ The University of Melbourne, United States

With recent improvements in resolution, brought about by advances in detectors [1], software reconstruction approaches [2] and advanced TEM grids [3], and the development of more automated cryoTEM workflows, attention now turns to specimen preparation as a remaining bottleneck to highthroughput solution of biological structures. To best ensure success in a high end cryo-TEM the user must first screen their sample to ensure the best possible sample purity, optimal particle concentration and ice thickness. Ice should be thin enough to encapsulate the protein but not much thicker since thick ice decrease the signal-to-noise ratio of the single particle images [4], deteriorating eventual resolution. Thus high-throughput cryo-TEM would benefit greatly from routine and high-throughput ice thickness measurement.

Currently there are a number of ways of measuring, or at least estimating, ice thickness. One can perform tomography on the area of interest, with the time-consuming acquisition of a tilt series and then reconstruction of the 3D volume [5]. Alternatively, one can measure the proportion of electrons inelastically scattered using a post-specimen energy filter and relate this to the ice thickness [6]. Finally, one can burn a hole into the ice, tilt the stage and estimate ice thickness from the geometry of the resulting ice "channel" [7]. An alternative, dubbed the "aperture limited scattering" (ALS) approach, estimates the thickness of ice from a TEM image of the grid with a post-specimen objective aperture inserted [6]. Thicker regions of ice appear darker in the image since in these regions more of the incident beam is scattered to higher angles and excluded from the image by the objective aperture. This technique is fast, requiring the acquisition of only a single image, requires only the most basic workhorse TEM and can simultaneously measure ice thickness over a few micron sized field of view in real time.

Currently the coefficient of proportionally that relates the image intensity to ice thickness must be calibrated for a given combination of TEM accelerating voltage and aperture size (in mrad) using one of the other methods for ice thickness measurement. In this work we show how these can be calculated from first principles simulations, see Fig 1. comparing ice thickness measurements from our simulation to experiment using the ice channel approach for our Thermo Fisher Talos L120C. An example ice thickness map for holes produced using this method is displayed in Fig 2. These simulations require that accurate physical models of multiple inelastic and elastic scattering and the correct amorphous structure of vitreous ice be considered.

We discuss progress towards developing an open-source, user-friendly tool for users to calculate image intensity vs ice thickness look-up tables for their own microscopes and to convert TEM images into ice thickness maps of their grids. The only barrier to a centre incorporating this method in their workflows is the measurement of their objective apertures in angular units (milliradians) using a standard cross grating sample or similar. 


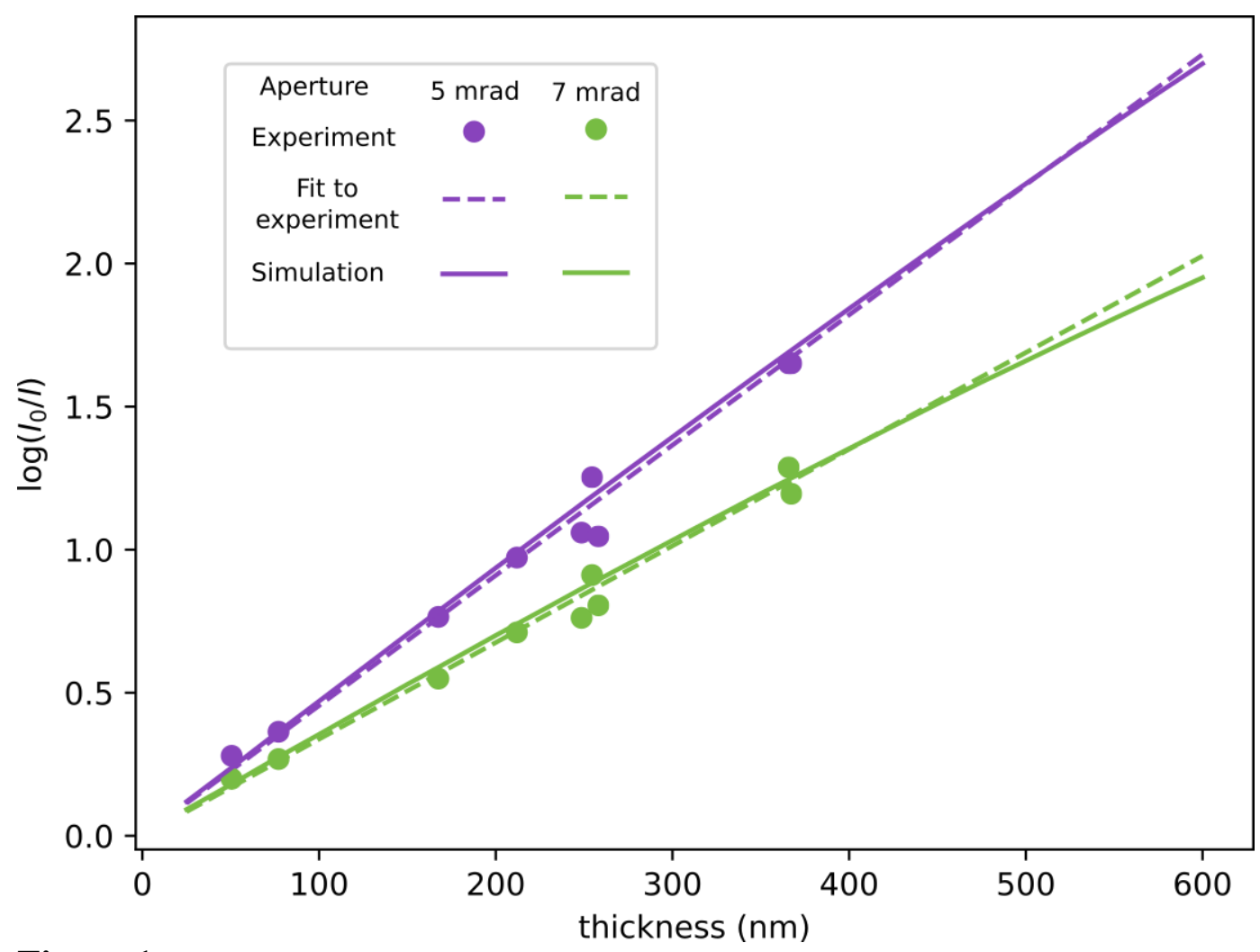

Figure 1. Simulated thickness-relative intensity curves for different apertures on a Thermo Fisher Talos L120C instrument $(120 \mathrm{kV}$ accelerating voltage) plotted with experimentally measured values for ice thickness using the "channel" measurement method
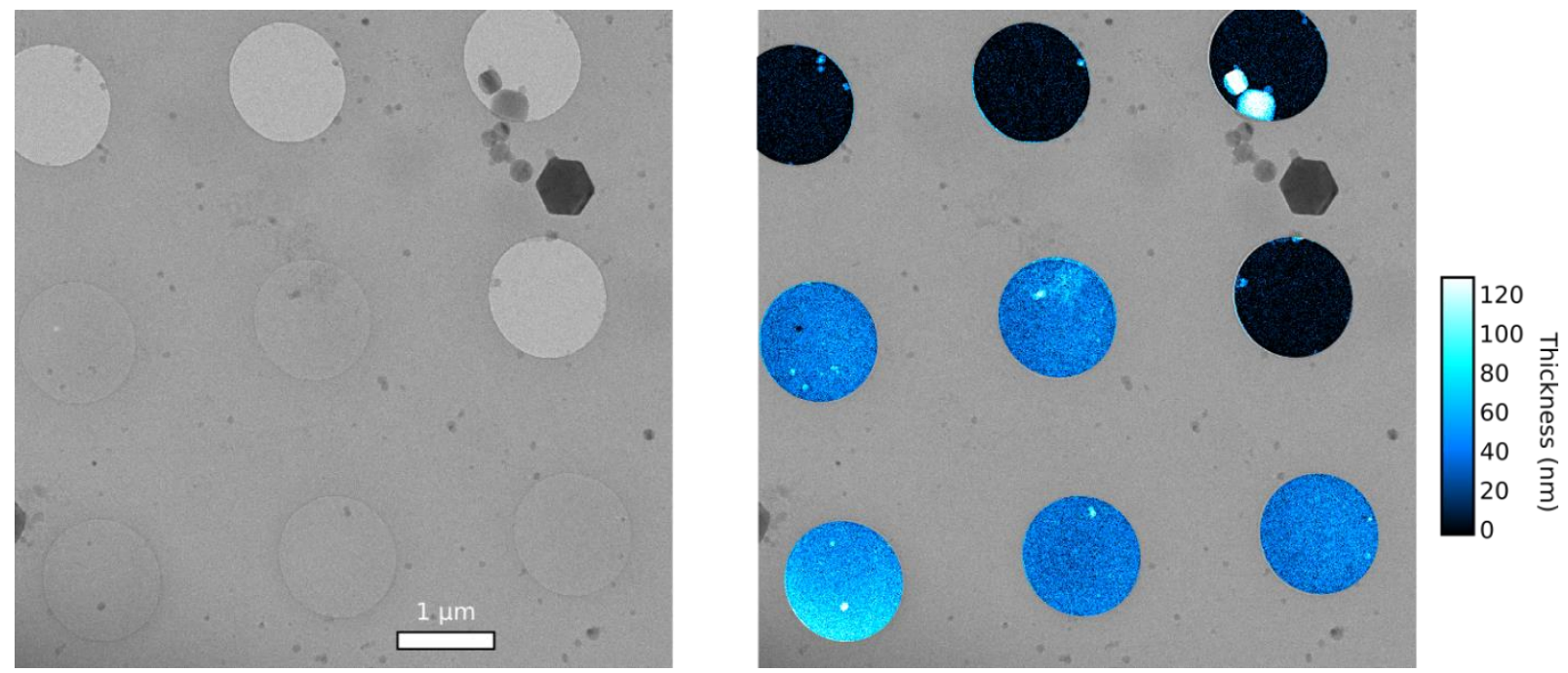

Figure 2. Ice thickness measurement on a quantifoil grid on a Thermo Fisher Arctica microscope at $200 \mathrm{kV}$, (a) is the raw micrograph and (b) has been overlaid with a map of the estimated ice thickness in each of the grid holes. Note that the method is inaccurate for contaminant ice crystallites.

\section{References}

[1] Subramaniam, Sriram, et al. "Resolution advances in cryo-EM enable application to drug discovery." Current opinion in structural biology 41 (2016): 194-202. 
[2] Zivanov, Jasenko, et al. "New tools for automated high-resolution cryo-EM structure determination in RELION-3." elife 7 (2018): e42166.

[3] Russo, Christopher J., and Lori A. Passmore. "Ultrastable gold substrates for electron cryomicroscopy." Science 346.6215 (2014): 1377-1380.

[4] Baxter, William T., et al. "Determination of signal-to-noise ratios and spectral SNRs in cryo-EM lowdose imaging of molecules." Journal of structural biology 166.2 (2009): 126-132.

[5] Noble, Alex J., et al. "Routine single particle CryoEM sample and grid characterization by tomography." Elife 7 (2018): e34257.

[6] Rice, William J., et al. "Routine determination of ice thickness for cryo-EM grids." Journal of structural biology 204.1 (2018): 38-44.

[7] Angert, Isabel, et al. "Elastic and inelastic scattering cross-sections of amorphous layers of carbon and vitrified ice." Ultramicroscopy 63.3-4 (1996): 181-192.

[8] We acknowledge use of the ThermoFischer Arctica and Tecnai F30 microscopes at the Ian Holmes Imaging centre, Bio21 Institute at the University of Melbourne 